\title{
Surface modification of titanium-coated glass substrate embedded acrylate-based hydrogel film for optical metal clad leaky waveguide (MCLW) biosensors
}

DOI:

10.1109/ICSENS.2017.8234289

\section{Document Version \\ Accepted author manuscript}

Link to publication record in Manchester Research Explorer

Citation for published version (APA):

Makhsin, S. R., Gardner, P., Goddard, N., \& Scully, P. (2017). Surface modification of titanium-coated glass substrate embedded acrylate-based hydrogel film for optical metal clad leaky waveguide (MCLW) biosensors. In IEEE Sensors 2017 (Vol. INSPEC Accession Number: 17434441). (IEEE Sensors Conference). IEEE. https://doi.org/10.1109/ICSENS.2017.8234289

\section{Published in:}

IEEE Sensors 2017

\section{Citing this paper}

Please note that where the full-text provided on Manchester Research Explorer is the Author Accepted Manuscript or Proof version this may differ from the final Published version. If citing, it is advised that you check and use the publisher's definitive version.

\section{General rights}

Copyright and moral rights for the publications made accessible in the Research Explorer are retained by the authors and/or other copyright owners and it is a condition of accessing publications that users recognise and abide by the legal requirements associated with these rights.

\section{Takedown policy}

If you believe that this document breaches copyright please refer to the University of Manchester's Takedown Procedures [http://man.ac.uk/04Y6Bo] or contact uml.scholarlycommunications@manchester.ac.uk providing relevant details, so we can investigate your claim.

\section{OPEN ACCESS}




\title{
Surface modification of titanium-coated glass
}

\section{substrate embedded acrylate-based hydrogel film for optical metal clad leaky waveguide (MCLW) biosensors}

\author{
Siti R. Makhsin ${ }^{1,2, *}$, P. Gardner ${ }^{1}$, Nicholas J. Goddard ${ }^{3}$, and Patricia J. Scully ${ }^{4}$ \\ ${ }^{1}$ School of Chemical Engineering and Analytical Science, Manchester Institute of Biotechnology, The University of \\ Manchester, 131 Princess Street, Manchester, M1 7DN, UK. \\ ${ }^{2}$ Faculty of Mechanical Engineering, Universiti Teknologi MARA, 40450 Shah Alam, Malaysia \\ ${ }^{3}$ Process Instruments (UK) Ltd, March Street, Burnley BB12 0BT, UK \\ ${ }^{4}$ School of Chemical Engineering and Analytical Science, The University of Manchester, The Mill Sackville Street, \\ Manchester, M13 9PL, UK \\ *sitirabizah.makhsin@manchester.ac.uk
}

\begin{abstract}
For the highly-sensitive optical MCLW biosensor, the stability of the acrylate-based hydrogel waveguiding sensing layer deposited onto the Ti (titanium)-coated glass substrate depends on the quality of surface modification between the interfaces. This study demonstrates an optimised silanization technique to form covalent bonds across the interface between the modified Ti-coated glass substrate and the deposited acrylate-based hydrogel waveguiding film, to prevent detachment. The optical characteristics of the MCLW device outcome depend on the refractive index changes on the cover medium. Hence, the device sensitivity fabricated on the surface modified substrate generated a strong signal at $\mathbf{2 5 0}$ A.U which is $45 \%$ better than previously reported agarosebased hydrogel MCLW sensor. The signal proved to have excellent stability throughout the detection for at least 3 hours without any background noise.
\end{abstract}

Keywords-metal clad leaky waveguide; optical biosensor; acrylate hydrogel; silanization

\section{INTRODUCTION}

The development of optical biosensors with high sensitivity and specificity is of remarkable interest for medical diagnostics and environmental monitoring, where rapid and real-time detection of the analyte such as bacteria, antigens, viruses, etc., is necessary [1]. Furthermore, the practical biosensors should also be robust and stable as the devices need to operate over longer test periods in wet conditions to detect the analyte. Robust detection requires that the sensor should operate without breakdown or failure over the required period. For optical biosensor including metal clad leaky waveguide (MCLW) device, the stability of the hydrogel waveguide sensing layer depends on the composition and alignment to the sensor surface, which has to be homogeneously supported on the entire substrate [2]. The substrate surface must be strongly covalently bound to the hydrogel to avoid detachment during operation.

In our previous study [3], a composition acrylate hydrogel made of PEGMEA (Mn 480) as a monomer, PEGDA (Mn 700) as a crosslinker and acrylate-PEG $\mathrm{P}_{2000^{-}}$ NHS (ACLT-PEG-NHS) as a linker had produced a single

The first author has been awarded an SLAI scholarship from Ministry of Higher Education Malaysia. mode waveguide of the signal transducer of MCLW spectroscopy (see Fig.1). The result showed that the acrylatebased hydrogel waveguiding layer had a high porosity thus promising to offer better sensitivity. However, weak adhesion binding of the deposited hydrogel on the substrate restricted the detection time to 1.5 hour after which the hydrogel film detached from the titanium-coated glass substrate due to swelling.

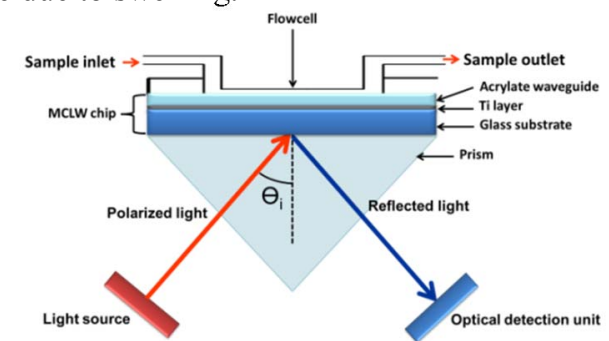

Fig. 1. Principle of optical MCLW device with acrylate-based hydrogel as a waveguiding sensing layer.

One approach to overcome this weakness is by modified the surface of the substrate using silanization technique as the silane layer ideally provide accessible binding moieties while at the same time can minimising nonspecific binding [2]. Silanization enables bond formation at the interface between the substrate and hydrogel deposited on the substrate, by adsorption, self-assembly and covalent binding of silane molecules onto substrate surfaces [2, 4]. The technique involved treating the surface of substrates with organofunctional alkoxysilane molecules such as methoxy ($\left.\mathrm{OCH}_{3}\right)$ and the ethoxy $\left(-\mathrm{OCH}_{2} \mathrm{CH}_{3}\right)$ [5]. The substrates either glass or metal oxides surfaces can be silanized because they consist of hydroxyl groups $(-\mathrm{OH})$ which attack and replace the alkoxy groups on the silane, to create a covalent $\mathrm{Si}-\mathrm{O}-\mathrm{Si}$ bond.

The motivation of this paper is to investigate surface modification mechanisms which promise to improve binding of the acrylate-based hydrogel thin film to the Ti-coated glass substrate. The objective includes to achieve a stable hydrogel film forming on the surface of the treated substrate 
that is resistant to washing by the solvent medium, thus the detection time can be prolonged by maintaining a stable waveguide sensing layer on the substrate during wetting and swelling of the hydrogel. The silanization includes using silyl functional group; 3-(trimethoxysilyl) propyl methacrylate; TMSPMA or aminosilanes; 3-aminopropyl-triethoxysilane; APTES to treat the substrate as shown in Fig.2 (a, b). Subsequently, ACLT-PEG-NHS is introduced into the silanization process (see Fig.2, c) after the substrate being treated with APTES. The addition of ACLT-PEG-NHS to treat the substrate is essential in order to create a covalent bond between $-\mathrm{NH}$ functional group with the acrylate-based hydrogel film. Moreover, the hydrophilicity of the treated substrate can be enhanced from the $-\mathrm{PEG}_{2000}$ compound thus improving the adhesion and homogeneity of hydrogel distribution on the substrate to produce a waveguide signal from the spin coating process.

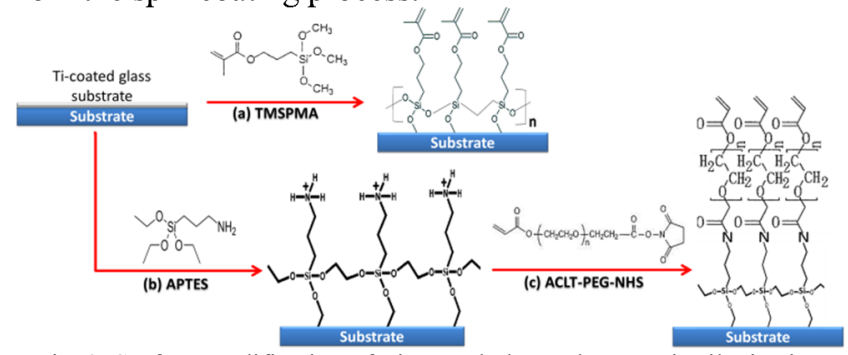

Fig. 2. Surface modification of Ti-coated glass substrate via silanization process using (a) TMSPMA, (b) APTES and (c) APTES and ACLT-PEGNHS.

\section{MATERIALS AND METHOD}

\section{A. Materials}

The materials used to fabricate the MCLW device are 1.2 mm thick glass slides (VWR, UK), titanium; Ti $(>99.99 \%$, 433667), 3-aminopropyl-triethoxysilane; APTES $(\geq 98 \%$, A3648), 3-(Trimethoxysilyl)propyl methacrylate; TMSPMA ( $\geq 98 \%$, M6514), PEG diacrylate; PEGDA (Mn700, 455008), PEG methyl ether acrylate; PEGMEA (Mn 480, 454990), HEPES sodium salt (H8651), sodium hydroxide; $\mathrm{NaOH}$ (99.99\%, 306576), 2-Hydroxy-4'-(2-hydroxyethoxy)-2methyl propiophenone; HHEMP (98\%, 410896), toluene (99.8\%, 244511) all: Sigma-Aldrich, UK. Acrylate-PEG ${ }_{2000}-$ NHS ester; (ZZ236P009) from JENKEM Technology, USA and glycerol, (>99.5\%, G10650108), Phosphate buffer saline; PBS (10X, P5493) and absolute ethanol (E/0650DF/17) bought from Fisher Scientific, UK.

\section{B. Methods and instrumentation}

At first, the glass slides were cleaned using 0.5\% Decon 90 followed by $0.1 \mathrm{M} \mathrm{NaOH}$ and finally ethanol in the ultrasonic water bath for 30 minutes each. The slides then rinsed with deionized (DI) water and dried in the oven at $110^{\circ} \mathrm{C}$ for at least 1 hour. Then, the glass slides were coated with titanium at the thickness $9.5 \mathrm{~nm}$ using e-beam deposition machine at $10^{-6} \mathrm{mbar}$ and rate of $0.16 \mathrm{mms}^{-1}$. The Ti-coated glass slides were cut into $2.5 \times 2.5 \mathrm{~cm}$ size using a diamond cutter. Silanization was performed by treating the Ti-coated glass substrate either using TMSPMA (Fig.2, a) or $5 \% \mathrm{v} / \mathrm{v}$ APTES prepared in absolute ethanol (Fig. $2, \mathrm{~b})$ for 1 hour. Then the treated substrate was rinsed and dried in the oven at $110^{\circ} \mathrm{C}$ for at least 1 hour. On the other hand, the substrate that has been silanized using 5\% v/v APTES was further incubated with $1 \% \mathrm{v} / \mathrm{v}$ ACLT-PEG-NHS (Fig. 2, c) for 2 hours at room temperature. The substrate then extensively rinsed with DI water and dried overnight in the oven until ready to use. In order to fabricate the MCLW chip, the $40 \% \mathrm{v} / \mathrm{v}$ of hydrogel made of $32.9 \% \mathrm{v} / \mathrm{v}$ PEGMEA, $6.09 \%$ v/v PEGDA, $0.33 \% \mathrm{w} / \mathrm{v}$ of HHEMP and $2.5 \% \mathrm{v} / \mathrm{v}$ of ACLT-PEG-NHS was added as an ester. Then, the solution mixture was placed in the ultrasonic water bath for 10 minutes. After that, the solution was dropped onto the treated substrate and incubated at room temperature for 15 minutes. After that, the chip was spun at $5500 \mathrm{rpm}$ or 6000 rpm or $6500 \mathrm{rpm}$ for three mins. The chips were further crosslinked to solidify the hydrogel using UV light (Dymax 2000-EC, EC $400 \mathrm{~W}, 105 \mathrm{mWcm}^{-2}$ ) for 10 minutes under nitrogen environment. Finally, the chip was attached to the flow cell and clamped onto the optical waveguide set-up as shown in Fig.1 to measure the refractive index (RI) change $(\Delta \mathrm{n})$. The fabrication of the chips has been repeated several times as each sample needs in freshly prepared for each testing. The details of the instrumentation setup used to test the device have been previously described $[3,6]$.

\section{RESULTS AND DISCUSSION}

The surface modification technique using silanization (step a and b in Fig.2.), generated only one sample with an acceptable optical waveguide signal. The functional sample was the MCLW chip formed from the Ti-coated glass substrate treated with APTES. No waveguide signal was generated by the chip formed from TMSPMA treated substrate since no hydrogel was deposited onto the substrate through the spin coating process. This was caused by the chip treated with TMSPMA (consisting of 3 groups of methoxy $\left(-\mathrm{OCH}_{3}\right)$, being very hydrophobic, causing the hydrogel to leave the substrate during the spin coating process. For the MCLW chip treated with APTES, the hydrogel spun at $6000 \mathrm{rpm}$, generated a single waveguide mode when tested as shown in the waveguide profile in Fig.3. Observation of a waveguided mode indicated that the hydrogel waveguiding film was thin enough to support a single mode of the waveguiding signal.

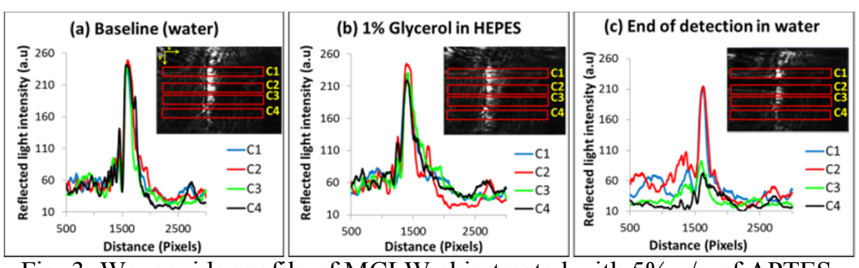

Fig. 3. Waveguide profile of MCLW chip treated with $5 \% \mathrm{v} / \mathrm{v}$ of APTES tested with different RI buffer (a) baseline with DI water, (b) 1\% Glycerol (c) rinse with water.

However, the inset image in Fig.3 shows that the intensity of the reflected light (shown as a white line) is not a uniform peak, even though a single mode of the waveguide was supported. Theoretically, the angular 
reflectance of the waveguide is affected by the thickness and RI of the hydrogel waveguiding material [4]. Higher waveguide modes were caused by increased thickness of the waveguiding film, controlled by the spin coater speed used to deposit the hydrogel on the treated substrate. The red boxes in this figure represent the area which detection of $\Delta \mathrm{n}$ will be monitored. Due to the unhomogenised light intensity of the signal throughout the sensor surface, only 4 test columns can be drawn as a label with $\mathrm{C} 1, \mathrm{C} 2, \mathrm{C} 3$, and $\mathrm{C} 4$. The comparison waveguide profile was plotted using measured data from column $\mathrm{C} 2$ to see the shift of the peak intensity for $\Delta \mathrm{n}$ measurement. The peak position of the reflected light intensity shifted to the left from 1600 pixels to 1300 pixels when the higher RI buffer (Fig.3, b) flowed over the cover medium, and the signal returned to the baseline pixels position when the test solution changed back to water (Fig.3, c). However, at the end of this detection sensitivity experiment (see peak Fig.3, c) the reflected light intensity reduced indicating that hydrogel detached from the sensor chip. The individual waveguide profile shows that the monitored signal (reflected light intensity) from column C3 and $\mathrm{C} 4$, decreases and then disappear from the detection area at the end of the detection sensitivity procedure. This indicates that the binding affinity between the hydrogel waveguiding layer and substrate is inadequate.
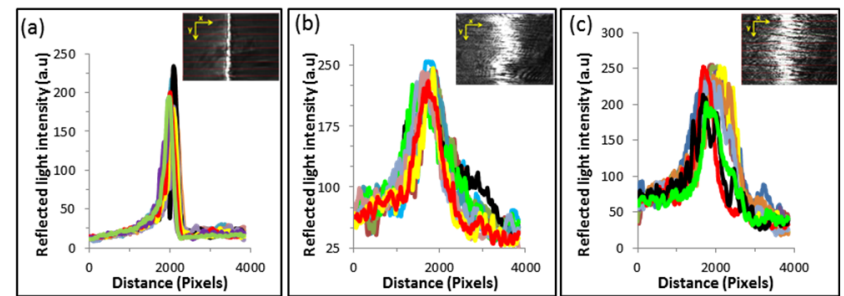

Fig. 4. Waveguide profile of the modified MCLW chips using APTES and ACLT-PEG-NHS fabricated by depositing at different spin speeds with (a) 5500 (b) 6000 and (c) $6500 \mathrm{rpm}$.

The ideal waveguide signal indicated when the chip was treated with $5 \% \mathrm{v} / \mathrm{v}$ of APTES followed with $1 \% \mathrm{v} / \mathrm{v}$ of ACLT-PEG-NHS as clearly observed Fig.4,a with hydrogel spun at $5500 \mathrm{rpm}$. The peak reflected light intensities at the test areas are identical, indicating that the hydrogel film was deposited at the right spin speed and thus thickness. The waveguide mode signal formed a single peak (see inset image). Moreover, all reflected light peaks (from 10 tested columns scanned throughout the inset image) were observed at the same position ( 2000 pixels) indicating that the hydrogel was homogeneously distributed with uniform thickness across the surface of the substrate, and supported a single guided mode without any noise. The ideal waveguide mode forms a thin just under cut-off thickness, peak and responds to changes during the detection, thus enhancing the sensitivity of the device. However, increasing the spin speeds to $6000 \mathrm{rpm}$ and $6500 \mathrm{rpm}$ to spin the same composition of hydrogel precursor does not improve the signal, as the waveguide mode is distorted, due to high spin speed forming a too thin film of the hydrogel. Fig.5 represents the waveguide profile of MCLW chip from the modified Ti coated substrate with $2.5 \% \mathrm{v} / \mathrm{v}$ of NHS linker spun at $5500 \mathrm{rpm}$ when a different RI buffer was used throughout the experiment. The reflected light intensity generated from the sample measured at $249.3 \pm 8.6$ A.U with the percent deviation is $3.5 \%$ (number of samples = 35 ). The entire column in the hydrogel layer producing an identical peak signal as clearly shown in Fig.4, a. The Moreover, the signal was $45 \%$ improved from the previously reported agarose-based MCLW sensor [6]. This sample was the only one to sustain an intact hydrogel layer at the end of the sensitivity test with the stable signal for at least 3 hours [3]. The waveguide mode retained single mode signal intensity (Fig.5, f), indicating that the hydrogel layer was strongly covalent bond with treated substrate with $2.5 \%$ NHS linker from the hydrogel precursor.

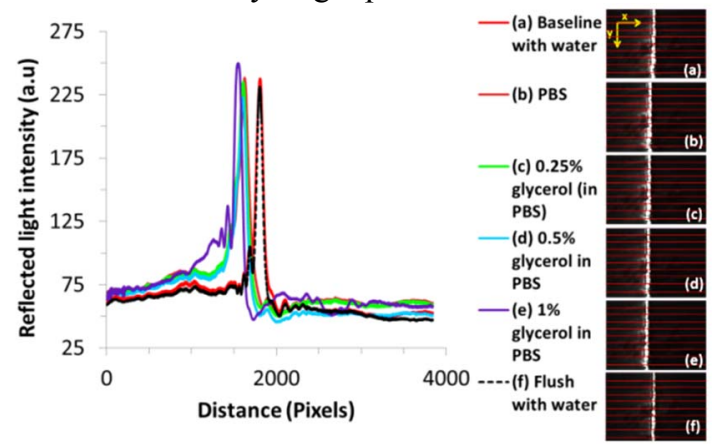

Fig. 5. Waveguide profile of MCLW chip made of Ti-coated glass substrate treated with $5 \% \mathrm{v} / \mathrm{v}$ of APTES and ACLT-PEG-NHS spun at $5500 \mathrm{rpm}$ tested with different RI buffer.

\section{CONCLUSIONS}

The optimisation procedure of silanization is to treat the substrate with $5 \% \mathrm{v} / \mathrm{v}$ of APTES followed by treating with $1 \%$ ACLT-PEG-NHS. The ideal signal of single waveguide mode was formed from the MCLW chip made of surface modified Ti-coated glass substrate with the hydrogel was spun at $5500 \mathrm{rpm}$ spin speed demonstrating enhanced sensitivity up to $45 \%$ and remain stable for more than 3 hours of the detection for biosensing application.

\section{ACKNOWLEDGMENT}

The authors greatly appreciated all the technical support from School of Chemical Engineering and Analytical Science, and Manchester Institute of Biotechnology of The University of Manchester.

\section{REFERENCES}

[1] H. K. Hunt, C. Soteropulos, and A. M. Armani, "Bioconjugation strategies for microtoroidal optical resonators," Sensors, vol. 10, no. 10, pp. 9317-9336, 2010.

[2] M. Lessel, O. Bäumchen, M. Klos et al., "Self-assembled silane monolayers: A step-by-step high speed recipe for high-quality, low energy surfaces," arXiv preprint arXiv:1212.0998, 2012.

[3] S. R. Makhsin, N. J. Goddard, R. Gupta et al., "Tailored synthesis of photopatternable acrylate-based hydrogel for optical metal clad leaky waveguide (MCLW) biosensors," unpublished, 2017.

[4] A. D. McNaught, and A. D. McNaught, Compendium of chemical terminology: Blackwell Science Oxford, 1997.

[5] B. Seed, "Silanizing glassware," Current Protocols in Cell Biology, pp. A. 3E. 1-A. 3E. 2, 2001.

[6] R. Gupta, and N. Goddard, "A novel optical biosensor with internal referencing." pp. 1490-1492. 